\title{
$K$-THEORY AND MULTIPLIERS OF STABLE $C^{*}$-ALGEBRAS
}

\author{
J. A. MINGO
}

\begin{abstract}
The main theorem is that if $A$ is a $C^{*}$-algebra with a countable approximate identity consisting of projections, then the unitary group of $M(A \otimes K)$ is contractible. This gives a realization, via the index map, of $K_{0}(A)$ as components in the set of Fredholm operators on $H_{A}$.
\end{abstract}

For a $C^{*}$-algebra $A$ let $H_{A}=\left\{\left(a_{i}\right) \mid a_{i} \in A\right.$ and $\sum a_{i}^{*} a_{i}$ converges in norm $\}$. Then $H_{A}$ becomes a right $A$-module under the action $\left(a_{i}\right) b=\left(a_{i} b\right)$. With the $A$-valued inner product $\left\langle\left(a_{i}\right),\left(b_{i}\right)\right\rangle=\sum a_{i}^{*} b_{i}, H_{A}$ is a Hilbert $A$-module. $\mathscr{L}\left(H_{A}\right)$ is the $C^{*}$-algebra of operators on $H_{A}$ that have an adjoint; $\mathscr{K}\left(H_{A}\right)$ is an ideal of $\mathscr{L}\left(H_{A}\right)$, called the compact operators on $H_{A}$. Set [11, §2, Definition 4]. Operators in $\mathscr{L}\left(H_{A}\right)$ invertible modulo $\mathscr{K}\left(H_{A}\right)$ are called Fredholm and have an index that takes its values in $K_{0}(A)$.

The paper is divided into two parts. In Part 1 we show that index: $\left[\mathscr{F}_{A}\right] \rightarrow K_{0}(A)$ is an isomorphism, where $\left[\mathscr{F}_{A}\right]$ denotes the set of path components of the set of Fredholm operators.

In Part 2 we show that the unitary group of $M(A \otimes K)$ is contractible when $A$ has a countable approximate identity consisting of projections. As $\mathscr{L}\left(H_{A}\right) \cong$ $M(A \otimes K)$, this in particular implies that the unitary group of $\mathscr{L}\left(H_{A}\right)$ is connected when $A$ is unital. This latter result is used to prove the isomorphism mentioned above.

The results of this paper were announced in [14] (see Remark 2.5(2)). This research is based on the author's doctoral work done at Dalhousie University under the supervision of Professor P. A. Fillmore. The author also wishes to thank W. J. Phillips for many useful suggestions.

\section{PART 1. THE INDEX OF A FREDHOLM OPERATOR}

1.1. We describe the index map from Fredholm operators on Hilbert $C^{*}$-modules to the corresponding $K$-groups. The principal ingredients of this construction have been discussed by several authors: Kasparov [12], Miščenko and Fomenko [15], and Pimsner, Popa, and Voiculescu [17]. However, as we shall need refinements and variations of these constructions, we will give a detailed account of the index map.

Throughout this paper $K$ or $\mathscr{K}(H)$ will denote the $C^{*}$-algebra of compact operators on a separable infinite dimensional Hilbert space $H$. For any two $C^{*}$-algebras $A$ and $B, A \otimes B$ will denote the completion of the algebraic tensor product $A \odot B$ in the spatial or minimal $C^{*}$-norm (see e.g. Effros and Lance [8, §2]).

Received by the editors July 28, 1983 and, in revised form December 27, 1985.

1980 Mathematics Subject Classification (1985 Revision). Primary 46L05, 46M20.

C1987 American Mathematical Society $0002-9947 / 87 \$ 1.00+\$ .25$ per page 
Regarding Hilbert $C^{*}$-modules we will follow the notation of Kasparov [11, §2]; if $\mathscr{E}$ and $\mathscr{F}$ are Hilbert $A$-modules, then $\mathscr{L}(\mathscr{E}, \mathscr{F})$ denotes the set of adjointable maps from $\mathscr{E}$ to $\mathscr{F}$, and $\mathscr{K}(\mathscr{E}, \mathscr{F})$ denotes the ideal in $\mathscr{L}(\mathscr{E}, \mathscr{F})$ consisting of compact operators.

$K_{0}(A)$ will denote the $K$-group of the $C^{*}$-algebra $A$ as defined in Taylor [19]. Murray-von Neumann equivalence of projections is denoted by " " and stable equivalence class by $[\cdot]$. When convenient (and appropriate) we will regard $K_{0}(A)$ as the Grothendieck group of the semigroup of isomorphism classes of finitely generated projective $A$-modules, where again stable equivalence will be denoted by [ ].

Definition. Let $\mathscr{E}$ and $\mathscr{F}$ be Hilbert $A$-modules and $T \in \mathscr{L}(\mathscr{E}, \mathscr{F}) . T$ is Fredholm if there exists $S \in \mathscr{L}(\mathscr{F}, \mathscr{E})$ with $1-S T \in \mathscr{K}(\mathscr{E}, \mathscr{E})$ and $1-T S \in$ $\mathscr{K}(\mathscr{F}, \mathscr{F})$.

The index map associates to a Fredholm operator $T \in \mathscr{L}(\mathscr{E})$, where $\mathscr{E}$ is a Hilbert $A$-module, an element, index $(T)$, of $K_{0}(A)$. This map generalizes the usual index map of Hilbert space theory in the following sense. If $H$ is an ordinary Hilbert space, then the usual inner product makes $H$ into a Hilbert $\mathbf{C}$-module and our construction will associate, to a Fredholm operator $T \in \mathscr{L}(H)$, index $(T)$ in $K_{0}(\mathbf{C})$. Now $K_{0}(\mathbf{C}) \cong \mathbf{Z}$ by the dimension map, and the usual index map is $T \mapsto$ $\operatorname{dim}($ index $T$ ).

Let $H_{A}$ be the Hilbert space over $A,\left[11, \S 2\right.$, Example 2]. Let $P_{n} \in \mathscr{L}\left(H_{A}\right)$ be the selfadjoint map which sends $\left(a_{1}, a_{2}, \ldots\right)$ to $\left(a_{1}, a_{2}, \ldots, a_{n}, 0, \ldots\right)$. Then $P$ is a projection and $P_{n} \xi \rightarrow \xi$ for all $\xi$ in $H_{A}$. Thus $P_{n} \mathcal{O}_{\xi, \eta} \rightarrow \mathcal{O}_{\xi, \eta}$ for all $\xi, \eta$ in $H_{A}$ (see [11, Lemma 3] for the definition of $\left.\mathcal{O}_{\xi, \eta}\right)$. Hence $P_{n} K, K P_{n} \rightarrow K$ for all $K \in \mathscr{K}\left(H_{A}\right)$. If $A$ is unital then $H_{A}$ has the standard orthonormal basis $\left\{\xi_{i}\right\}_{1}^{\infty}$ where $\xi_{i}$ is the sequence with zeros everywhere but the $i$ th place, where there is a 1 . Then $P_{n}=\sum_{i=1}^{n} \mathcal{O}_{\xi_{i}, \xi_{i}} \in \mathscr{K}\left(H_{A}\right)$ and so $\left\{P_{n}\right\}_{n=1}^{\infty}$ forms an approximate identity for $\mathscr{K}\left(H_{A}\right)$, consisting of projections.

1.2. We give, without proof, the version of a standard lemma that will be convenient for subsequent use.

Lemma. Let $B$ be a $C^{*}$-algebra. Suppose $e$ and $f$ are projections in $B$ and $\| f-$ fef $\| \leqslant \delta<1 / 18$. Then there exists a unitary in $B$ (or $\tilde{B}$ if $B$ does not have a unit) with $u f u^{*} \leqslant e$ and $\|u-1\| \leqslant 18 \delta$.

1.3. If $A$ is unital and $P$ is a projection in $\mathscr{K}\left(H_{A}\right)$ with $P \leqslant P_{n}$ for some $n$, then the range of $P$ is a complemented submodule of $A^{(n)}$ and so is finitely generated and projective. By $A^{(n)}$ we mean the free $A$-module of rank $n$, and we regard $A^{(n)}$ as the submodule of $H_{A}: A^{(n)}=\left\{\left(a_{i}\right) \mid 9_{i}=0, i>n\right\}$. By Lemma 1.2 every projection in $\mathscr{K}\left(H_{A}\right)$ is equivalent to a $P \leqslant P_{n}$. Therefore we have

Lemma [12, §6, Lemma 5]. Suppose that $A$ is unital. The range of a projection in $\mathscr{K}\left(H_{A}\right)$ is a finitely generated and projective $A$-module.

A simple observation used here and later is that if $P$ and $Q$ are projections in $\mathscr{L}\left(H_{A}\right)$, then $P \sim Q$ as projections implies that their ranges are isomorphic as $A$-modules. 
1.4. Suppose that $A$ is unital and that $F \in \mathscr{L}\left(H_{A}\right)$ is a Fredholm operator. We would like to take $[\operatorname{ker} F]-\left[\operatorname{ker} F^{*}\right]$ to be the definition of index $F$. However, for many such $F$ 's $\operatorname{ker} F$ and $\operatorname{ker} F^{*}$ may fail to be finitely generated.

Definition. Let $F \in \mathscr{L}\left(H_{A}\right)$ be Fredholm. If $G \in \mathscr{L}\left(H_{A}\right)$ has finitely generated kernel and cokernel and closed range and $G-F \in \mathscr{K}\left(H_{A}\right)$, then define

$$
\operatorname{index}(F)=[\operatorname{ker} G]-\left[\operatorname{ker} G^{*}\right] \in K_{0}(A) .
$$

In the next few paragraphs we will show that such a $G$ always exists and the element of $K_{0}(A)$ so defined depends only on $F$.

1.5. Proposition (Pimsner, Popa, and Voiculescu [17, Lemma 7.4]). Let $U \in \mathscr{L}\left(H_{A}\right)$ be unitary modulo $\mathscr{K}\left(H_{A}\right)$. Then for $n$ large enough $U\left(1-P_{n}\right)$ has a polar decomposition, i.e. there is a partial isometry $V$ in $\mathscr{L}\left(H_{A}\right)$ with $U\left(1-P_{n}\right)=$ $V\left|U\left(1-P_{n}\right)\right|$. Such a $V$ is a compact perturbation of $U$.

Proof. Choose $n$ large enough that $\left\|\left(1-P_{n}\right)\left(1-U^{*} U\right)\left(1-P_{n}\right)\right\|<1$ and let

$$
V=U\left(1-P_{n}\right)\left(P_{n}+\left(1-P_{n}\right) U^{*} U\left(1-P_{n}\right)\right)^{-1 / 2}
$$

1.6. Lemma (cf. [12, §6, Lemma 5]). Suppose that $A$ is unital. If $\mathscr{E} \subseteq H_{A}$ is a finitely generated closed submodule of $H_{A}$, then there is a projection $P$ in $\mathscr{K}\left(H_{A}\right)$ whose range is $\mathscr{E}$.

Proof. Let $\left\{\eta_{i}\right\}_{i=1}^{n}$ be a set of generators for $\mathscr{E}$. Let $K=\sum_{i=1}^{n} \mathcal{O}_{\eta_{i}, \xi_{i}}$ where $\left\{\xi_{i}\right\}$ is the standard orthonormal basis for $H_{A}$ (see Definition 1.1). Then $K\left(H_{A}\right)=\mathscr{E}$ and $K\left(A^{(n)}\right)=\mathscr{E}$, where we regard $A^{(n)}$ as the submodule of $H_{A}:\left\{\left(a_{1}, a_{2}, \ldots\right) \mid a_{i}=0\right.$, $i \geqslant n+1\}$. Now $\||K| \eta\|=\|K \eta\|$ for all $\eta$, and as $\mathscr{E}$ is closed, we have that $|K|\left(A^{(n)}\right)$ is closed.

Next we claim that $|K| \mathscr{K}\left(A^{(n)}\right)$ is a closed subset of $\mathscr{K}\left(A^{(n)}\right)$. This follows because $\mathscr{K}\left(A^{(n)}\right) \cong M_{n}(A) \cong A^{(n)} \oplus \cdots \oplus A^{(n)}$. Therefore by [3, Lemma III.2.9, p. 335] there is a projection $P$ in $\mathscr{K}\left(A^{(n)}\right)$ such that $|K| A^{(n)}=P A^{(n)}$ and $|K| P=|K|$. Then $|K|$ is invertible in $P \mathscr{K}\left(A^{(n)}\right) P=\mathscr{K}\left(P A^{(n)}\right)$. Denote its inverse by $|K|^{-1}$. We may regard $P \in \mathscr{K}\left(A^{(n)}\right)$ as an operator on $H_{A}$ by making it zero on $A^{(n) \perp}=$ $\left(1-P_{n}\right) H_{A}$; similarly, we regard $|K|^{-1}$ as an operator on $H_{A}$ by making it zero on $(1-P) H_{A}$. Now let $V=K|K|^{-1}$. Then $V$ is a partial isometry in $\mathscr{K}\left(H_{A}\right)$ with range $\mathscr{E}$. So $V V^{*}$ is the desired projection.

1.7. Proposition. Suppose that $A$ is unital and $F \in \mathscr{L}\left(H_{A}\right)$. If $F$ is Fredholm then there is $G$, a compact perturbation of $F$, with closed range and finitely generated kernel and cokernel.

Proof. Choose $U \in \mathscr{L}\left(H_{A}\right)$ so that $\pi(U)=\pi(F) \pi(|F|)^{-1}$ where $\pi: \mathscr{L}\left(H_{A}\right) \rightarrow$ $\mathscr{L}\left(H_{A}\right) / \mathscr{K}\left(H_{A}\right)$ is the quotient map. Then by Proposition 1.5 there is $V$, a partial isometry, for which $U-V \in \mathscr{K}\left(H_{A}\right)$. Now choose $H$ selfadjoint such that $\pi(H)=$ $\log \pi(|F|)$. Then $R=e^{H}$ is positive and invertible and $G=V R$ is a compact perturbation of $F$. Now $\operatorname{range}(G)=\operatorname{range}(V)$ which is closed, and $\operatorname{ker} G=\operatorname{ker} V=$ $\operatorname{range}\left(1-V^{*} V\right)$, and $\operatorname{ker} G^{*}=\operatorname{ker} V^{*}=\operatorname{range}\left(1-V V^{*}\right)$ which are finitely generated by 1.3 . 
REMARKS. (1) It can be shown that any operator $F \in \mathscr{L}\left(H_{A}\right)$ with closed range and finitely generated kernel and cokernel is Fredholm. This uses an argument of W. J. Phillips which shows that as $F$ has closed range there is $V$ in $\mathscr{L}\left(H_{A}\right)$, a partial isometry, with $\operatorname{range}(V)=\operatorname{range}(F), \operatorname{ker}(V)=\operatorname{ker}(F)$, and $F=V|F|$. If $P$ is the projection onto $\operatorname{ker} F$, then $P \in \mathscr{K}\left(H_{A}\right)(1.6)$ and $|F|+P$ is invertible. Also by 1.6, $1-V^{*} V$ and $1-V V^{*} \in \mathscr{K}\left(H_{A}\right)$, so $V$ is unitary modulo $K\left(H_{A}\right)$. Hence $F$ is invertible modulo $\mathscr{K}\left(H_{A}\right)$; that is, $F$ is Fredholm.

(2) Notice that the proof showed slightly more than was claimed. Namely, given a Fredholm operator $F$ we may find a compact perturbation $G$ with closed range and finitely generated kernel and cokernel so that $\operatorname{ker} G=P_{n}\left(H_{A}\right)$, where $P_{n}$ is as in 1.1 and $V$ is chosen as it is in Proposition 1.5.

1.8. We have now shown the existence part in the definition of the index map in 1.4. Now we will show that it is well defined. This means showing that if $F$ and $G$ are Fredholm, $F-G \in \mathscr{K}\left(H_{A}\right)$, and have closed range and finitely generated kernel and cokernel then

$$
[\operatorname{ker} F]-\left[\operatorname{ker} F^{*}\right]=[\operatorname{ker} G]-\left[\operatorname{ker} G^{*}\right]
$$

in $K_{0}(A)$. This easily reduces to the case where $F$ and $G$ are partial isometries; write (by 1.7) $F=V|F|, G=U|G|$. Then $\pi(U) \pi(|F|)=\pi(V) \pi(|G|)$ implies that $U-V$ $\in \mathscr{K}\left(H_{A}\right)$. Then since $\operatorname{ker} U=\operatorname{ker} F, \operatorname{ker} U^{*}=\operatorname{ker} F^{*}$, etc., we are reduced to the case when $F$ and $G$ are partial isometries.

Lemma [12, §6, TheOrem 3 PRoOF of unAmbiguity]. Let $U$ and $V$ be Fredholm partial isometries with $U-V \in \mathscr{K}\left(H_{A}\right)$. Then $[\operatorname{ker} U]-\left[\operatorname{ker} U^{*}\right]=[\operatorname{ker} V]-$ $\left[\operatorname{ker} V^{*}\right]$ in $K_{0}(A)$.

This completes the definition of the index map. The set of Fredholm operators on $H_{A}$ is denoted by $\mathscr{F}_{A} . \mathscr{F}_{A}$ is a semigroup under multiplication. When $A$ is unital we have a map: index: $\mathscr{F}_{A} \rightarrow K_{0}(A)$. In the next few paragraphs we will establish some of the expected properties for this map-that it is a locally constant surjective homomorphism.

1.9. Proposition [12, §6, Lemma 1]. The map index: $\mathscr{F}_{A} \rightarrow K_{0}(A)$ is locally constant.

Proof. Let $F \in \mathscr{F}_{A}$ and choose $\varepsilon>0$ so that $\|F-G\|<\varepsilon$ implies that

$$
\left\|\pi(F) \pi(|F|)^{-1}-\pi(G) \pi(|G|)^{-1}\right\|<1 .
$$

Then choose $U$ and $V$ such that

$$
\pi(U)=\pi(F) \pi(|F|)^{-1}, \quad \pi(V)=\pi(G) \pi(|G|)^{-1}
$$

and $\|U\|,\|U-V\|<\sqrt{2}$. Letting $X=2-U^{*} U+U^{*} V$ and $Y=2-U U^{*}+$ $U V^{*}$ we have that $\|2-X\|,\|2-Y\| \leqslant\|U\|\|U-V\|<2$, and so $X$ and $Y$ are invertible.

Now index $(F)=\operatorname{index}(U)$, for if $F_{0}=W\left|F_{0}\right|$ is a compact perturbation of $F$ with $W$ a partial isometry then $W$ is a compact perturbation of $U$. Similarly index $G=\operatorname{index} V$. As $X$ and $Y$ are invertible, we have $\operatorname{index}(U X)=\operatorname{index} U$ and 
index $(Y V)=\operatorname{index} V$, as $\operatorname{ker}(W X)=X^{-1} \operatorname{ker}(W)$. Finally index $(U X)=\operatorname{index}(Y V)$ because

$$
U X-Y V=\left(1-U U^{*}\right)(U-V)+U\left(1-V V^{*}\right)-\left(1-U^{*} U\right) V \in K\left(H_{A}\right) .
$$

As index $(F \oplus G)=\operatorname{index} F+\operatorname{index} G$ and there is a path of Fredholm operators connecting $F \oplus G$ to $F G \oplus 1$ (see e.g. [12, § Lemma 2] or [17, Lemma 7.5]), we have

Corollary. The map index: $\mathscr{F}_{A} \rightarrow K_{0}(A)$ is a homomorphism.

1.10. To show that the index map is onto, we begin with an observation of Atiyah [2, Appendix]. If $p \in A$ is a projection and $S \in \mathscr{L}(H)$ is an isometry, then $x=(1-p) \otimes 1+p \otimes S$ is an isometry in $A \odot \mathscr{L}(H)$ with $1-x x^{*}=p \otimes(1-$ $\left.S S^{*}\right)$.

Lemma. Suppose that $A$ is unital and $p \in A \otimes K$ is a projection. Then there is an isometry $w$ in $A \otimes \mathscr{L}(H)$ with $1-w w^{*}=p$.

Proof. We may assume that $A \subseteq \mathscr{L}\left(H_{0}\right)$ for some Hilbert space $H_{0}$; then $A \otimes K$ and $A \otimes \mathscr{L}(H)$ may be defined to be the closures of the subalgebras of $\mathscr{L}\left(H_{0} \otimes H\right)$ generated by $\{a \otimes k \mid a \in A, k \in \mathscr{K}(H)\}$ and $\{a \otimes b \mid a \in A, b \in \mathscr{L}(H)\}$, respectively.

Let $\left\{e_{i j}\right\}$ be a set of matrix units for $\mathscr{K}(H)$. Let $p_{n}=1 \otimes \sum_{i=1}^{n} e_{i i} \in A \otimes K$. Then $\left\{p_{n}\right\}_{1}^{\infty}$ is an approximate identity for $A \otimes K$.

Suppose first that $p \leqslant p_{n}$ for some $n$. Then $p=\sum_{i, j=1}^{n} a_{i j} \otimes e_{i j}$ for some $a_{i j} \in A$. Let $U: H \rightarrow \mathbf{C}^{n} \otimes H$ be a unitary which carries $e_{i j}$ to $f_{i j} \otimes e_{11}($ for $i, j=1, \ldots, n$ ) where $\left\{f_{i j}\right\}$ is a set of matrix units for $\mathscr{L}\left(\mathbf{C}^{n}\right)$. Then $1 \otimes U: H_{0} \otimes H \rightarrow H_{0} \otimes \mathbf{C}^{n} \otimes$ $H$ carries $p$ to a projection of the form $p^{\prime} \otimes e_{11}$, where $p^{\prime}=\sum_{i, j=1}^{n} a_{i j} \otimes f_{i j}$. By Atiyah's observation there is an isometry $x \in A \otimes M_{n} \otimes \mathscr{L}(H)$ such that $1-x x^{*}$ $=p^{\prime} \otimes e_{11}$. Then $v=(1 \otimes U)^{*} x(1 \otimes U)$ is an isometry in $A \otimes \mathscr{L}(H)$ with $1-$ $v v^{*}=p$.

If $p \in A \otimes K$ is arbitrary, then by choosing $n$ large enough we have $\left\|p-p_{n} p p_{n}\right\|$ $<1 / 18$. So by Lemma 1.2 there is a unitary $U$ in $(A \otimes K)^{\sim}$ with $U^{*} p U \leqslant p_{n}$. If $1-v v^{*}=U^{*} p U$ as above then $w=U W U^{*}$ is an isometry in $A \otimes \mathscr{L}(H)$ with $1-w w^{*}=p$.

COROllary. Let $P \in \mathscr{K}\left(H_{A}\right)$ be a projection. Then there is an isometry $W$ in $\mathscr{L}\left(H_{A}\right)$ with $P=1-W W^{*}$. Moreover index: $\mathscr{F}_{A} \rightarrow K_{0}(A)$ is onto.

Proof. $M(A \otimes K)$ denotes the multiplier algebra of $A \otimes K$ (see [6]). Since $A \otimes K$ is an essential ideal in $A \otimes \mathscr{L}(H)$, we may regard $A \otimes \mathscr{L}(H)$ as a subalgebra of $M(A \otimes K)$ [6, Proposition 3.7]. By [11, Theorem 1] there is an isomorphism of $M(A \otimes K)$ onto $\mathscr{L}\left(H_{A}\right)$ which sends $A \otimes K$ to $\mathscr{K}\left(H_{A}\right)$. To prove the first claim, apply the lemma above.

To see that the index map is onto let $\mathscr{E}, \mathscr{F} \subseteq A^{(n)}$ be finitely generated projective $A$-modules. Let $P, Q \in M_{n}(A)$ be the projections of $A^{(n)}$ onto $\mathscr{E}$ and $\mathscr{F}$, respectively (where, if necessary, we replace $\mathscr{E}$ and $\mathscr{F}$ by isomorphic copies to assure the selfadjointness of $P$ and $Q)$. Since $A^{(n)} \cong P_{n}\left(H_{A}\right)$ and $M_{n}(A) \cong P_{n} \mathscr{K}\left(H_{A}\right) P_{n}$, we will regard $P$ and $Q$ as projections in $\mathscr{K}\left(H_{A}\right)$. So choose isometries $V$ and $W$ with 
$1-V V^{*}=P$ and $1-W W^{*}=Q$. Then

$$
\operatorname{index}\left(W V^{*}\right)=\left[\operatorname{ker} W V^{*}\right]-\left[\operatorname{ker} V W^{*}\right]=[\mathscr{E}]-[\mathscr{F}] \text {. }
$$

1.11. Proposition [17, Lemma 7.5]. If $F \in \mathscr{F}_{A}$ and index $(F)=0$, then there is $G$ in $\mathscr{L}\left(H_{A}\right)$ invertible with $F-G \in \mathscr{K}\left(H_{A}\right)$.

Proof. We may suppose that $F$ has closed range and its kernel and cokernel are finitely generated. Let $F=V|F|$ be the polar decomposition of $F$ given in Proposition 1.7. Moreover by Remark 2 following Proposition 1.7 we may suppose that $\operatorname{ker}(F)=P_{n}\left(H_{A}\right)$ for some $n$. Now index $F=0$ means $P_{n}\left(H_{A}\right)$ is stably isomorphic to $\operatorname{ker} F^{*}$. So let $\mathscr{E}$ be a finitely generated and projective $A$-module for which

$$
P_{n}\left(H_{A}\right) \oplus \mathscr{E} \cong \operatorname{ker} F^{*} \oplus \mathscr{E} .
$$

As before we may suppose that there is a projection $P$ in $\mathscr{K}\left(H_{A}\right)$ with $P \leqslant P_{n+m}-$ $P_{n}$ and $\mathscr{E} \cong P\left(H_{A}\right)$

Let $V_{1}=V(1-P)$. The $V_{1}$ is a partial isometry with $\operatorname{ker} V_{1}=P_{n}\left(H_{A}\right)+P\left(H_{A}\right)$ and $\operatorname{ker} V_{1}^{*}=\operatorname{ker} V^{*}+V(\mathscr{E}) \cong \operatorname{ker} F^{*} \oplus \mathscr{E}$. So there is a partial isometry $W$ in $\mathscr{K}\left(H_{A}\right)$ with $W^{*} W=\operatorname{ker} V_{1}$ and $W W^{*}=\operatorname{ker} V_{1}^{*}$. Thus $G=\left(V_{1}+W\right)\left(|F|+P_{n}\right)$ is invertible and $G-F \in \mathscr{K}\left(H_{A}\right)$.

1.12. The index map just constructed has a less explicit version that is easier to construct. If $O(A \otimes K)$ denotes the quotient algebra $M(A \otimes K) / A \otimes K$, then the index map is the connecting homomorphism $\delta: K_{1}(O(A \otimes K)) \rightarrow K_{0}(A \otimes K)$. As observed by J. Cuntz $K_{*}(M(A \otimes K))=\{0\}$, so $\delta$ is an isomorphism. If $V$ is a Fredholm partial isometry in $\mathscr{L}\left(H_{A}\right)$, then $V=\pi(V)$ is unitary and

$$
\begin{aligned}
\delta(v) & =\left[\left(\begin{array}{ll}
V & 1-V V^{*} \\
V^{*} V-1 & V^{*}
\end{array}\right)\left(\begin{array}{ll}
1 & 0 \\
0 & 0
\end{array}\right)\left(\begin{array}{ll}
V^{*} & V^{*} V-1 \\
1-V V^{*} & V
\end{array}\right)\right]-\left[\left(\begin{array}{ll}
1 & 0 \\
0 & 0
\end{array}\right)\right] \\
& =\left[\left(\begin{array}{ll}
1-V^{*} V & 0 \\
0 & 0
\end{array}\right)\right]-\left[\left(\begin{array}{ll}
1-V V^{*} & 0 \\
0 & 0
\end{array}\right)\right] \\
& =\operatorname{index}(V) .
\end{aligned}
$$

The advantage of using the index map constructed above is that it gives us more explicit information about Fredholm operators: in the next section we will see that two Fredholm operators have the same index precisely when they are in the same path component of $\mathscr{F}_{A}$.

When $A$ is not unital, the index construction given here does not work, mainly because $A \otimes K$ may fail to have any projections, and when this happens there are no Fredholm partial isometries in $\mathscr{L}\left(H_{A}\right)$. One way around this is to use the stabilization theorem [11], when $A$ has a strictly positive element. If $A$ does have a strictly positive element, then $H_{A}$ is a countably generated Hilbert $\tilde{A}$-module, so $H_{A} \oplus H_{\tilde{A}} \cong H_{\tilde{A}}$. Then send $T \in \mathscr{L}\left(H_{A}\right)$ to $T \oplus I$ in $\mathscr{L}\left(H_{A} \oplus H_{\tilde{A}}\right) \cong \mathscr{L}\left(H_{\tilde{A}}\right)$. If $T$ is Fredholm, then so is $T \oplus I$. Now we can obtain index $(T \oplus I) \in K_{0}(\tilde{A})$. In fact,

$$
\text { index }(T \oplus I) \in \operatorname{ker} \varepsilon_{*} \equiv K_{0}(A) \subseteq K_{0}(\tilde{A})
$$

where $\varepsilon: \tilde{A} \rightarrow \mathrm{C}$ is the augmentation map. As noted above, Proposition 1.7 is not true in the nonunital case in general. This leaves the question: When $A$ is nonunital but has a strictly positive element if $T \in \mathscr{L}\left(H_{A}\right)$ is Fredholm and $\operatorname{index}(T)=0$, then is there $K \in \mathscr{K}\left(H_{A}\right)$ so that $T+K$ is invertible? 
The index map obeys the expected functoriality relations. If $\varphi: A \rightarrow B$ is a unital * homomorphism then $\tilde{\varphi}=\varphi \otimes \mathrm{id}_{K}: A \otimes K \rightarrow B \otimes K$ is nondegenerate (i.e. $\tilde{\varphi}(A \otimes K) \cdot B \otimes K$ is dense in $B \otimes K)$ so we have a unital extension $\tilde{\varphi}: M(A \otimes K)$ $\rightarrow M(B \otimes K)$ which carries $\mathscr{F}_{A}$ into $\mathscr{F}_{B}$. As expected we have index $(\tilde{\varphi}(T))=$ $\varphi_{*}$ index $(T)$ as is easily verified by checking when $T$ is a partial isometry.

The index map respects Kasparov's product. Suppose $A$ and $B$ are unital and $F \in \mathscr{L}\left(H_{A}\right), G \in \mathscr{L}\left(H_{B}\right)$ are Fredholm with polar decomposition $F=V|F|, G=$ $U|G|$. Let $F^{\prime}=|F|^{-1} V^{*}, G^{\prime}=|G|^{-1} U^{*}$ where the inverses are taken on $\operatorname{ker} F^{\perp}$ and $\operatorname{ker} G^{\perp}$ respectively. Unwinding the formula in [12, §4, Remark 3] ( $A$ and $B$ are trivially graded) we have

$$
F \# G=\left(\begin{array}{cc}
\left(1-V^{*} V\right) \otimes G & F^{\prime} \otimes 1 \\
F \otimes 1 & \left(V V^{*}-1\right) \otimes G^{\prime}
\end{array}\right) \in \mathscr{L}\left(H_{A} \otimes H_{B} \oplus H_{A} \otimes H_{B}\right) .
$$

Let $W$ be the partial isometry

$$
\left(\begin{array}{cc}
\left(1-V^{*} V\right) \otimes U & V^{*} \otimes 1 \\
V \otimes 1 & \left(V V^{*}-1\right) \otimes U^{*}
\end{array}\right) .
$$

Then $F \# G=W|F \# G|$. And so

$$
\operatorname{index}(F \# G)=\operatorname{index} F \otimes \operatorname{index} G \in K_{0}(A \otimes B)
$$

as

$$
\begin{aligned}
{\left[1-W^{*} W\right] } & =\left[\begin{array}{cc}
1-V^{*} V \otimes 1-U^{*} U & 0 \\
0 & 1-V V^{*} \otimes 1-U U^{*}
\end{array}\right] \\
& =[\operatorname{ker} F \otimes \operatorname{ker} G]+\left[\operatorname{ker} F^{*} \otimes \operatorname{ker} G^{*}\right]
\end{aligned}
$$

and

$$
\begin{aligned}
{\left[1-W W^{*}\right] } & =\left[\begin{array}{cc}
1-V^{*} V \otimes 1-U U^{*} & 0 \\
0 & 1-V V^{*} \otimes 1-U^{*} U
\end{array}\right] \\
& =\left[\operatorname{ker} F \otimes \operatorname{ker} G^{*}\right]+\left[\operatorname{ker} F^{*} \otimes \operatorname{ker} G\right] .
\end{aligned}
$$

1.13. To conclude Part 1 we will show that for any compact Hausdorff space $X$, $\left[X, \mathscr{F}_{A}\right]$, the set of homotopy equivalence classes of maps of $X$ into $\mathscr{F}_{A}$, forms a group isomorphic to $K_{0}(C(X) \otimes A)$ via the index map. Our first step is to prove this when $X$ is a point; in this case $\left[X, \mathscr{F}_{A}\right]$ will just be denoted by $\left[\mathscr{F}_{A}\right]$.

Proposition. Suppose that $A$ is unital. Then with the operation of multiplication $\left[\mathscr{F}_{A}\right]$ is a group and index: $\left[\mathscr{F}_{A}\right] \rightarrow K_{0}(A)$ is an isomorphism.

Proof. We saw in Proposition 1.9 that index is constant on paths so we have a well-defined map index: $\left[\mathscr{F}_{A}\right] \rightarrow K_{0}(A)$. We give $\left[\mathscr{F}_{A}\right]$ the obvious operation $[F][G]$ $\equiv[F G]$. By Corollary $1.9 \operatorname{index}[F][G]=\operatorname{index}[F]+\operatorname{index}[G]$. Thus index is a homomorphism of semigroups which is surjective by Corollary 1.10. It is clear also that [1] is the identity of $\left[\mathscr{F}_{A}\right]$.

The remainder of the proposition will follow if we can show that index $[T]=0$ implies that $[T]=[1]$. To do this we need the main result of Part 2: the group of invertible elements of $M(A \otimes K) \cong \mathscr{L}\left(H_{A}\right)$ is connected. 
Now suppose $[T] \in\left[\mathscr{F}_{A}\right]$ with index $[T]=0$. Then by Proposition 1.11 there is $K \in \mathscr{K}\left(H_{A}\right)$ such that $V=T+K$ is invertible. So $t \mapsto T+t K, t \in[0,1]$, is a path of Fredholm operators connecting $T$ to $V$. Now by Theorem 2.5 there is a path of invertible (hence Fredholm) operators connecting $V$ to 1 .

If $[T] \in\left[\mathscr{F}_{A}\right]$, then index $[T]=-$ index $\left[T^{*}\right]$. Therefore index $[T]\left[T^{*}\right]=0$ and so $[T]\left[T^{*}\right]=\left[T T^{*}\right]=[1]$. Thus $\left[\mathscr{F}_{A}\right]$ is a group and index is an isomorphism.

Recall the isomorphism [11, Theorem 1] of $\mathscr{L}\left(H_{A}\right)$ with $M(A \otimes K)$. Also if $A$ and $B$ are any $C^{*}$-algebras, then $M(A) \otimes M(B)$ will be regarded as a subalgebra of $M(A \otimes B)[6$, Proposition 3.7].

Lemma. Suppose that $A$ and $B$ are unital. Then $\left[\mathscr{F}_{A \otimes B} \cap(A \otimes M(B \otimes K))\right]$ is a group and the inclusion map i: $\mathscr{F}_{A \otimes B} \cap(A \otimes M(B \otimes K)) \rightarrow \mathscr{F}_{A \otimes B}$ induces an isomorphism

$$
i_{*}:\left[\mathscr{F}_{A \otimes B} \cap(A \otimes M(B \otimes K))\right] \rightarrow\left[\mathscr{F}_{A \otimes B}\right]
$$

Proof. Obviously $i_{*}$ is a well-defined homomorphism of semigroups. If $T \in \mathscr{F}_{A \otimes B}$ $\cap(A \otimes M(B \otimes K))$ and $T$ may be connected to 1 in $\mathscr{F}_{A \otimes B}$, then index $T=0$. By Proposition 1.11 there is $K \in \mathscr{K}\left(H_{A \otimes B}\right) \cong A \otimes B \otimes K$ so that $V=T+K \in A \otimes$ $M(B \otimes K)$ is invertible. As before, $T$ may be connected to $V$ in $\mathscr{F}_{A \otimes B} \cap(A \otimes$ $M(B \otimes K))$. Now by Theorem 2.5 the group of invertible elements of $A \otimes$ $M(B \otimes K)$ is connected, so $V$ may be connected to 1 in $\mathscr{F}_{A \otimes B} \cap(A \otimes M(B \otimes K))$.

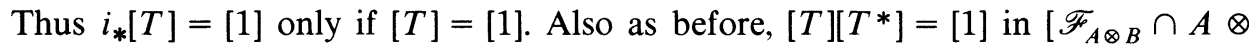
$M(B \otimes K)]$, and so $\left[\mathscr{F}_{A \otimes B} \cap(A \otimes M(B \otimes K))\right]$ is a group and $i_{*}$ is one-to-one.

To see that $i_{*}$ is onto, we must, given $T \in \mathscr{F}_{A \otimes B}$, find $S \in \mathscr{F}_{A \otimes B} \cap(A \otimes$ $M(B \otimes K))$ so that $[S]=[T]$, But by the Proposition this comes from showing that index $S=$ index $T$. By Lemma 1.10 and the argument used in the corollary which follows Lemma 1.10 there is $S \in A \otimes B \otimes \mathscr{L}(H) \subseteq A \otimes M(B \otimes K)$ with index $S$ $=\operatorname{index} T$.

THEOREM. For $A$ unital and $X$ compact and Hausdorff $\left[X, \mathscr{F}_{A}\right]$ is a group isomorphic to $K_{0}(C(X) \otimes A)$.

Proof. Identify $\left[X, \mathscr{F}_{A}\right]$, in the natural way, with

$$
\left[\mathscr{F}_{C(X) \otimes A} \cap C(X) \otimes M(A \otimes K)\right] .
$$

Then apply the lemma and we have

$$
\begin{aligned}
{\left[X, \mathscr{F}_{A}\right] } & \equiv\left[\mathscr{F}_{C(X) \otimes A} \cap(C(X) \otimes M(A \otimes K))\right] \\
& \stackrel{i_{*}}{\cong}\left[\mathscr{F}_{C(X) \otimes A}\right] \stackrel{\text { index }}{\cong} K_{0}(C(X) \otimes A) .
\end{aligned}
$$

COROllaRY. For $A$ unital $\pi_{n}\left(\mathscr{F}_{A}\right) \cong K_{n}(A)\left(\equiv K_{0}\left(C_{0}\left(\mathbf{R}^{n}\right) \otimes A\right)\right)$. 
Proof. Apply the five lemma to the diagram

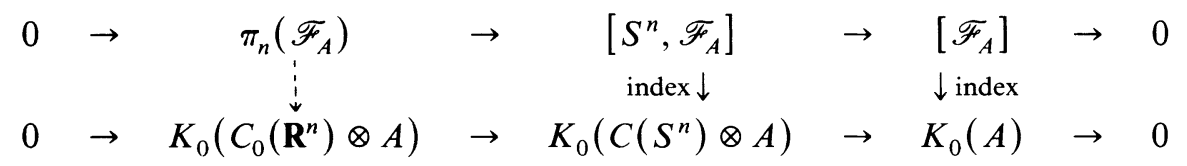

where the horizontal maps come from inclusion and evaluation at the north pole respectively.

\section{PART 2. CONTRACTIBILITY OF UNITARY GROUPS}

In this part we consider the question of the connectivity of the unitary group of $M(A \otimes K)$ and related algebras. Whenever we speak of a path of unitaries we mean a function from $[0,1]$ into $M(A \otimes K)$ which is norm continuous and unitary valued. The question of the connectivity of the unitary group of $M(A \otimes K)$ in the strict topology is solved in $[12, \S 1$, paragraph 17]. The main result is that the unitary group of $M(A \otimes K)$ is contractible (norm topology) when $A$ is unital or has a countable approximate identity consisting of projections.

2.1. LEMMA.

(a) Let $A$ be a $C^{*}$-algebra and $\left\{e_{i}\right\}_{i=1, \ldots}$, be a sequence of orthogonal projections in $M(A)$ such that $\sum_{i=1}^{\infty} e_{i}$ converges strictly [6, Definition 3.4]. Suppose $\left\{a_{i}\right\}$ is $a$ sequence in $M(A)$ which is bounded and satisfies $a_{i} e_{i}=e_{i} a_{i}=a_{i}$. Then $\sum_{i=1}^{\infty} a_{i}$ converges strictly and $\left\|\sum_{i=1}^{\infty} a_{i}\right\|=\sup _{i}\left\|a_{i}\right\|$.

(b) Suppose that $B$ is a unital $C^{*}$-algebra and that the $\left\{e_{i}\right\} \subseteq M(A)$ are as in (a) but in addition are pairwise equivalent via the partial isometries $s_{i j}: s_{i j} s_{j i}=e_{i}$. Then for $x \in B \otimes M(A)$

$$
\sum\left(1 \otimes s_{i 1}\right) x\left(1 \otimes s_{1 i}\right) \in B \otimes M(A) .
$$

Proof. The proof of (a) is clear. The point of (b) is that $\sum\left(1 \otimes s_{i 1}\right) x\left(1 \otimes s_{1 i}\right)$ is a strictly convergent series in $M(B \otimes A)$ but $B \otimes M(A)$ is only a norm closed subalgebra. However

$$
\left\|\sum\left(1 \otimes s_{i 1}\right) x\left(1 \otimes s_{1 i}\right)-\sum\left(1 \otimes s_{i 1}\right) y\left(1 \otimes s_{1 i}\right)\right\| \leqslant\|x-y\|
$$

for $x, y \in M(B \otimes A)$. So it suffices to show that $\sum_{i=1}^{\infty}\left(1 \otimes s_{i 1}\right) x\left(1 \otimes s_{1 i}\right) \in B \otimes$ $M(A)$ for $x$ in the algebraic tensor product $B \odot M(A)$. To achieve this we may suppose that $x=b \otimes a$ with $b \in B$ and $a \in M(A)$. In this case

$$
\sum_{i \equiv 1}^{\infty}\left(1 \otimes s_{i 1}\right) x\left(1 \otimes s_{1 i}\right)=b \otimes \sum_{i \equiv 1}^{\infty} s_{i 1} a s_{1 i} \in B \odot M(A) .
$$

This gives us the following straightforward generalization of [13, Lemma 7].

Proposition. Let $A$ and $B$ be $C^{*}$-algebras with $B$ unital. Let $u$ be a unitary in $B \otimes M(A)$. Suppose that $\left\{e_{i}\right\}$ is a sequence of orthogonal equivalent projections in $1_{B} \otimes M(A)$, with $\sum e_{i}=1$ strictly. If $u e_{i}=e_{i} u=e_{i}$ for $i=2,3,4, \ldots$, then $u$ may be connected to 1 by a path of unitaries in $B \otimes M(A)$. 
Proof. Let $v=e_{1} u e_{1}$. By $u \sim w$, we will denote that there is a path of unitaries connecting $u$ to $w$. The idea of the proof can be exhibited by the following diagram.

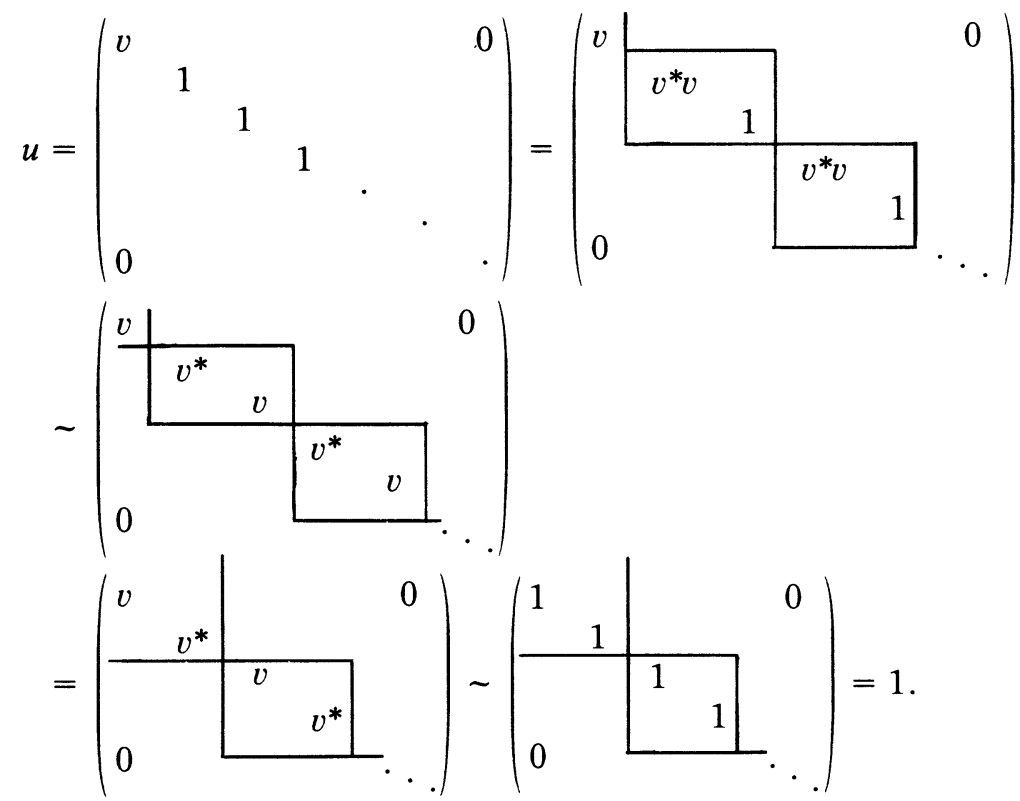

To make this rigorous we will describe the path giving the first " "; the second " " is handled similarly.

Let $\left\{s_{i j}\right\}$ be a family of partial isometries implementing the equivalences of the $\left\{e_{i}\right\}: s_{i j} s_{j i}=e_{i}$. Let $w_{i}=s_{i 1} v s_{1 i}$. By

$$
\left(\begin{array}{ccccc}
v & & & & 0 \\
& 1 & & & \\
& & 1 & & \\
0 & & & 1 & \ddots
\end{array}\right) \text { and }\left(\begin{array}{lllll}
v & & & & 0 \\
& v^{*} & & & \\
& & v & & \\
0 & & & v^{*} & \\
0 & & & &
\end{array}\right)
$$

we mean of course $v+e_{2}+e_{3}+\cdots$ and $v+w_{2}^{*}+w_{3}+w_{4}^{*}+\cdots$, respectively. By Lemma 2.1 the first series converges to $u$ and the second to a unitary, $w$, in $B \otimes M(A)$.

To connect $u$ to $w$ we use the standard path involving sines and cosines:

$$
s_{n}(t)=e_{2 n} \cos t-s_{2 n, 2 n+1} \sin t+s_{2 n+1,2 n} \sin t+e_{2 n+1} \cos t
$$

for $t \in[0, \pi / 2]$, Let, for $t \in[0, \pi / 2]$.

$$
\begin{aligned}
u(t)= & \left(v+\sum_{n=1}^{\infty}\left(w_{2 n}^{*}+e_{2 n+1}\right)\right) \\
& \cdot\left(e_{1}+\sum_{n=1}^{\infty} s_{n}(t)\right)\left(e_{1}+\sum_{n=1}^{\infty}\left(w_{2 n}+e_{2 n+1}\right)\right)\left(e_{1}+\sum_{n=1}^{\infty} s_{n}(t)\right)^{*}
\end{aligned}
$$

By Lemma 2.1, $e_{1}+\sum_{n=1}^{\infty} s_{n}(t)$ converges to a norm continuous path of unitaries in $1_{B} \otimes M(A)$ and both of $v+\sum_{n=1}^{\infty} w_{2 n}^{*}+e_{2 n+1}$ and $e_{1}+\sum_{n=1}^{\infty} w_{2 n}+e_{2 n+1}$ converge to unitaries in $B \otimes M(A)$. Now $u(0)=u$ and $u(\pi / 2)=w$. 
2.3. The next lemma is essentially contained in [13, Lemma 3] but more explicitly in $[4, \S 3.1]$.

Lemma. Let $A$ be a $C^{*}$-algebra with 1 and $e, f \in A$ orthogonal equivalent projections. If $u$ is a unitary in $A$ with $u^{*}$ eu orthogonal to $f$, then there is a path of unitaries in A connecting $u$ to a unitary $u_{1}$ satisfying $u_{1} e=e u_{1}=e$.

Proof. Let $v$ be a partial isometry with $v^{*} v=e$ and $v v^{*}=f$. Then $s=v+v^{*}+$ $1-e-f$ is a symmetry and thus may be connected to 1 , e.g. by

$$
s(x)=\exp (i(1-s) x) \text { for } x \in[0, \pi / 2] .
$$

Now $v u$ is a partial isometry with $(v u)^{*}(v u)=u^{*} e u$ and $(v u)(v u)^{*}=f$ so $t=v u+$ $(v u)^{*}+1-u^{*} e u-f$ is also a symmetry which may be connected to 1 . Thus $u_{1}=u t s$ is a unitary connectible to $u$ and $u_{1} e=e u_{1}=e$.

2.4. Lemma (Elliott [9]). Let $B$ be a $C^{*}$-algebra with unit and $A$ an ideal of $B$. Suppose that $B$ has a countable approximate identity consisting of projections, $\left\{e_{i}\right\}$. Let $u$ in $B$ be unitary. Then there is $v$ in $B$, unitary, such that $\|u-v\|<2$ and for some subsequence $\left\{f_{i}\right\}$ of $\left\{e_{i}\right\}$ we have

$$
v f_{1} v^{*}, v^{*} f_{1} v \leqslant f_{2} \leqslant v f_{3} v^{*}, v^{*} f_{3} v \leqslant f_{4} \leqslant \cdots \text {. }
$$

Proof. In the proof of [9, Theorem 2.4] Elliott proves the following. If $A$ is a separable matroid $C^{*}$-algebra with $\left\{e_{i}\right\} \subseteq A$, an approximate identity consisting of projections, then for every unitary $u$ in $M(A)$ there is a unitary $v$ with $\|u-v\|<2$ and a subsequence of $\left\{e_{i}\right\}$, which is again denoted $\left\{e_{i}\right\}$, such that $v e_{1} v^{*} \leqslant e_{2} \leqslant$ $v e_{3} v^{*} \leqslant e_{4} \cdots$. All that was used in the proof was that $A$ is an ideal in $M(A)$ having an approximate identity consisting of projections. Thus replacing $M(A)$ by $B$ we obtain a unitary $v$ in $B$ with $\|u-v\|<2$ and a subsequence $\left\{e_{i}\right\}$ such that $v e_{1} v^{*} \leqslant e_{2} \leqslant v e_{3} v^{*} \leqslant e_{4} \leqslant \cdots$. Now let $f_{i}=e_{2 i-1}$ for $i=1,2,3, \ldots$ and we are done.

2.5. Theorem Suppose that $A$ and $B$ are $C^{*}$-algebras with $B$ unital and $A$ having $a$ countable approximate identity consisting of projections (in particular, $A$ may be unital $)$. Then the unitary group of $B \otimes M(A \otimes K)$ is connected.

Proof. Let $u \in B \otimes M(A \otimes K)$ be a unitary. Let $\left\{f_{i}\right\} \subseteq A,\left\{g_{i}\right\} \subseteq K$ be countable approximate identities for $A$ and $K$ respectively consisting of projections. Let $e_{i}=1_{B} \otimes f_{i} \otimes g_{i}, i=1,2,3, \ldots ;$ when $A$ is unital let $e_{i}=1_{B} \otimes 1_{A} \otimes g_{i}$. Then $\left\{e_{i}\right\} \subseteq B \otimes A \otimes K$ is a countable approximate identity consisting of projections. We may apply Lemma 2.4 to obtain $v$ in $B \otimes M(A \otimes K)$ with $\|u-v\|<2$ and a subsequence of $\left\{e_{i}\right\}$, which will be denoted $\left\{e_{i}\right\}$, such that $v e_{1} v^{*}, v^{*} e_{1} v \leqslant e_{2} \leqslant$ $v e_{3} v^{*}, v^{*} e_{3} v \leqslant e_{4} \leqslant \cdots$. By standard methods (see e.g. [9, Theorem 2.4]) $u$ may be connected to $v$, so now our task is to connect $v$ to 1 .

Let $e=\sum_{i=0}^{\infty} e_{4 i+1}-e_{4 i}$ and $f=\sum_{i=0}^{\infty} e_{4 i+3}-e_{4 i+2}$, making the convention that $e_{0}=0$. By Lemma 2.1 these series converge strictly to projections in $1_{B} \otimes$ $M(A \otimes K)$. The inequalities $v^{*} e_{4 n+1} v \leqslant e_{4 n+2}$ and $e_{4 n-1} \leqslant v^{*} e_{4 n} v$ imply that $v^{*}\left(e_{4 n+1}-e_{4 n}\right) v \leqslant e_{4 n+2}-e_{4 n-1}$. Therefore $v^{*}\left(e_{4 n+1}-e_{4 n}\right) v$ is orthogonal to $e_{4 m+3}-e_{4 m+2}$ for $m, n=1,2,3, \ldots$. Thus both $e$ and $v^{*} e v$ are orthogonal to $f$. 
Next we want to show that $e, 1-e$, and $f$ are all equivalent to 1 in $1_{B} \otimes$ $M(A \otimes K)$. When $A$ is unital this is clear for then, by construction, $e, 1-e$, and $f$ are infinite projections in $1_{B} \otimes 1_{A} \otimes \mathscr{L}(H)$. Now in general

$$
\begin{aligned}
& e=1_{B} \otimes \sum_{n=0}^{\infty}\left(f_{4 n+1} \otimes g_{4 n+1}-f_{4 n} \otimes g_{4 n}\right), \\
& f=1_{B} \otimes \sum_{n=0}^{\infty}\left(f_{4 n+3} \otimes g_{4 n+3}-f_{4 n+2} \otimes g_{4 n+2}\right),
\end{aligned}
$$

and

$$
1-e=1_{B} \otimes \sum_{n=1}^{\infty}\left(f_{4 n} \otimes g_{4 n}-f_{4 n-3} \otimes g_{4 n-3}\right) .
$$

We will show that $e$ is equivalent to 1 ; the proof for $f$ and $1-e$ is similar.

We have to show that $\sum_{n=1}^{\infty}\left(f_{4 n+1} \otimes g_{4 n+1}-f_{4 n} \otimes g_{4 n}\right) \sim 1$ in $M(A \otimes K)$. Let $h_{n}=f_{n}-f_{n-1}$. Now for $k=[n / 4]$

$$
\begin{aligned}
&\left(h_{n} \otimes 1\right) \sum_{m=0}^{\infty}\left(f_{4 m+1}\right.\left.\otimes g_{4 m+1}-f_{4 m} \otimes g_{4 m}\right) \\
&=\left\{\begin{array}{l}
h_{n} \otimes \sum_{m \geqslant k+1}\left(g_{4 m+1}-g_{4 m}\right), \quad n \neq 1 \bmod 4, \\
h_{n} \otimes\left(g_{4 k+1}+\sum_{m \geqslant k+1} g_{4 m+1}-g_{4 m}\right), \quad n \equiv 1 \bmod 4, \\
=
\end{array}\right. \\
& h_{n} \otimes k_{n}
\end{aligned}
$$

where $k_{n}$ is an infinite projection in $\mathscr{L}(H)$. Let $v_{n}$ in $\mathscr{L}(H)$ be a coisometry with $v_{n}^{*} v_{n}=k_{n}$. Then $\sum_{n=1} h_{n} \otimes v_{n}$ converges strictly to a coisometry in $M(A \otimes K)$, as $\sum h_{n}$ converges strictly to 1 in $M(A)$. Let $x=1 \otimes \sum h_{n} \otimes v_{n}$, then

$$
\begin{aligned}
x^{*} x & =1 \otimes \sum h_{n} \otimes v_{n}^{*} v_{n}=1 \otimes \sum h_{n} \otimes k_{n} \\
& =1 \otimes \sum\left[\left(h_{n} \otimes 1\right) \sum_{m=0}^{\infty}\left(f_{4 m+1} \otimes g_{4 m+1}-f_{4 m} \otimes g_{4 m}\right)\right] \\
& =1 \otimes\left(\sum h_{n} \otimes 1\right) \sum_{m=0}^{\infty}\left(f_{4 m+1} \otimes g_{4 m+1}-f_{4 m} \otimes g_{4 m}\right) \\
& =e .
\end{aligned}
$$

Now we have $e, f$, and $1-e$ all equivalent to 1 . As $v^{*} e v$ is orthogonal to $f$ we may apply Lemma 2.3 to conclude that $v$ may be connected to a unitary $w$ with $w e=e w=e$.

Finally as $1_{A} \otimes \mathscr{L}(H) \subseteq M(A \otimes K)$ and $e \sim 1$, there is an orthogonal sequence of projections $\left\{d_{i}\right\}_{i=2}^{\infty}$ in $M(A \otimes K)$, each equivalent to 1 , with $\sum_{i=2} d_{i}=e$. Let $d_{1}=1-e$. We may now apply Proposition 2.2 to conclude that $w$ may be connected to 1 . Since we have shown that every unitary in $B \otimes M(A \otimes K)$ may be connected to 1 , the theorem is now proved. 
Corollary. If $A$ and $B$ are as in the theorem, then the unitary group of $B \otimes$ $M(A \otimes K)$ is contractible.

PROOF. The proof of the corollary uses the standard method and thus requires the following facts (see [13, §4] or [4, §4, Theorems 2 and 3]).

(1) If $X$ is an open subset of a Banach space and all the homotopy groups of $X$ are trivial, then $X$ is contractible.

(2) For any unital $C^{*}$-algebra $A, x \mapsto x|x|^{-1}$ is a retraction of the set of invertible elements, $\mathscr{G}$, onto the set of unitary elements, $\mathscr{U}$, which is homotopic to the identity map from $\mathscr{G}$ to $\mathscr{G}$.

Thus the homotopy groups of $\mathscr{U}$ and $\mathscr{G}$ are the same, and so by (1) $\mathscr{U}$ is contractible iff $\mathscr{G}$ is contractible.

So we must show that any unitary valued map from the $n$-sphere $S^{n}$ to $B \otimes$ $M(A \otimes K)$ with $f(*)=1$ (* is the north pole of $S^{n}$ ) is homotopic to the constant map: $x \mapsto 1$. Equivalently we must show that any unitary $f$ in $C\left(S^{n}\right) \otimes B \otimes$ $M(A \otimes K)$ with $f(*)=1$ can be connected to 1 by a path $f_{t}$ satisfying $f_{t}(*)=1$. By the theorem there is a path of unitaries, $f_{t}$, connecting $f$ to 1 and by replacing $f_{t}$ by $x \mapsto f_{t}(x) f_{t}(*)^{-1}$ we may suppose that $f_{t}(*)=1$ for all $t$.

ReMARKS. (1) As $(A \otimes K)^{\prime \prime}=A^{\prime \prime} \bar{\otimes} \mathscr{L}(H)$ for any $C^{*}$-algebra $A$, the conclusion of the corollary might be expected from the results of Breuer [4] and Brüning and Willgerodt [5], which show that the unitary group of $(A \otimes K)^{\prime \prime}$ is contractible. However, their methods of applying Lemma 2.3 are quite different; in the $W^{*}$ case one finds the projections to fit the unitary whereas, in the $C^{*}$ case, one adjusts the unitary to fit the projections. Also since tensoring with $C\left(S^{n}\right)$ takes an algebra out of the category of von Neumann algebras; in [4 and 13] it is necessary to connect a large number of unitaries to 1 simultaneously. There is only slight overlap between our results and the $W^{*}$ ones; for unital algebras $A, M(A \otimes K)$ is a von Neumann algebra only when $A$ is finite dimensional. For if $A$ is infinite dimensional, then there is a sequence of positive elements $\left\{x_{i}\right\}$ in $A$ with $\left\|x_{i}\right\|=1$ and $x_{i} x_{j}=0$ for $i \neq j$. Let $\left\{e_{i j}\right\} \subseteq K$ be a set of matrix units with $\sum e_{i i}=1$. Then $x=\sum x_{i} \otimes e_{i 1}$ converges weakly in $(A \otimes K)^{\prime \prime}$ but it is not a multiplier of $A \otimes K$, as

$$
\left\|\left(1-\sum_{i=1}^{n} 1 \otimes e_{i}\right) x\left(1 \otimes e_{1}\right)\right\|=\left\|\sum_{i=n+1}^{\infty} x_{i}\right\|=1
$$

instead of going to zero as it would if $x$ were a multiplier of $A \otimes K$.

(2) Theorem 3 in [14] is not correct. The subalgebra $1 \otimes \mathscr{L}(H)+A \otimes K$ of $M(A \otimes K)$ is strictly infinite but its unitary group is not in general connected ( $A$ is assumed unital). The problem is that the conclusion of Lemma 2.1(b) does not hold and so Kuiper's argument (Proposition 2.2) cannot be applied. By applying the $K$-theory six term exact sequence to the algebra $1 \otimes \mathscr{L}(H)+A \otimes K$ and ideal $A \otimes K$ we get an injection of $K_{1}(A)$ into $K_{1}(1 \otimes \mathscr{L}(H)+A \otimes K)$. Thus $K_{1}(1 \otimes \mathscr{L}(H)+A \otimes K)$ is not trivial in general and so the unitary group of $1 \otimes \mathscr{L}(H)+A \otimes K$ is not connected. The theorem above seems to be the strongest replacement for [14, Theorem 3]. 
(3) If one considers the Banach algebra $B \mathscr{L}\left(H_{A}\right)$, which consists of all bounded $A$-linear operators on $H_{A}$ [15], one can also show that the group of invertible elements of $B \mathscr{L}\left(H_{A}\right)$ is contractible. The proof is similar to that for $\mathscr{L}\left(H_{A}\right)$ except for the following modifications. It is necessary to work with invertible elements instead of unitaries; this is mainly an inconvenience as we have no control over $\left\|x^{-1}\right\|$. It is again only necessary to prove the connectedness of the group of invertible elements of $C\left(X, B \mathscr{L}\left(H_{A}\right)\right)$ - the Banach algebra of continuous functions from $X$ to $B \mathscr{L}\left(H_{A}\right)$ with the sup norm. In Lemma 2.4 we say $e \leqslant f$ if $e f=f e=e$ for idempotents $e$ and $f$ in a Banach algebra.

2.6. Theorem 2.5 suggests the question: for an $\mathrm{AF} C^{*}$-algebra when is $\mathscr{U}$, the unitary group of $M(A)$, contractible? In [9] Elliott showed that $\mathscr{U}$ is always connected (the proof is nominally for matroid $C^{*}$-algebras but works for AF $C^{*}$-algebras as well).

However, $\mathscr{U}$ is not always contractible; there are known obstructions to the triviality of $\pi_{1}(\mathscr{U})$ [1, Theorem $3.2 ; 7$, Lemma 5 , §VI]. Namely if $\tau$ is a bounded, everywhere-defined, normalized trace on $A$, which then extends to $M(A)$, then

$$
\chi_{\tau}: u_{t} \mapsto \frac{1}{2 \pi i} \int_{0}^{1} \tau\left(\frac{d u_{t}}{d t} u_{t}^{*}\right) d t
$$

is a homotopy invariant homomorphism from the piecewise smooth loops in $\mathscr{U}$ to $\mathbf{C}$, and thus defines a homomorphism $\chi_{\tau}: \pi_{i}(\mathscr{U}) \rightarrow \mathrm{C}$. Furthermore if $p$ is a projection in $M(A)$, then $\chi_{\tau}\left(e^{t 2 \pi i p}\right)=\tau(p)$, so $\chi_{\tau}$ is nontrivial.

Thus whenever there is a (finite) trace on $A, \pi_{1}(\mathscr{U})$ is nontrivial. The 0-dimensionality of $\hat{A}$ would suggest that $\pi_{0}$ and $\pi_{1}$ are the only obstructions to the contractibility of $\mathscr{U}$; in fact this is true.

THEOREM. Let $A$ be an $A F C^{*}$-algebra. Then the unitary group of $M(A)$ is contractible iff there are no (finite) traces on $A$.

Proof. By [10, Proposition 9.3] $A$ is stable iff there are no finite traces on $A$. So the theorem follows from Theorem 2.5.

2.8. It is still unknown to the author whether the unitary group of $M(A \otimes K)$ is connected for arbitrary $C^{*}$-algebras $A$. $A$ reasonable restriction might be that $A$ have a strictly positive element. Some results are known: when $A$ is separable and commutative or when $A=\Gamma_{0}(\mathscr{A})$ is a separable continuous trace $C^{*}$-algebra such that $\hat{A}$ has a finite cover which trivializes $\mathscr{A}$.

NOTE ADDED IN PROOF. It has recently been shown by J. Cuntz and N. Higson that if $A$ has a strictly positive element, then the unitary group of $M(A \otimes K)$ is contractible. Their paper, Kuiper's theorem for Hilbert modules will appear in Operator Algebras and Mathematical Physics, Contemporary Mathematics, Vol. 60, Amer. Math. Soc., Providence, R. I., 1986.

\section{REFERENCES}

1. H. Araki, M-S. B. Smith and L. Smith, On the homotopical significance of the type of a von Neumann algebra, Comm. Math. Phys. 22 (1971), 71-88.

2. M. Atiyah, K-theory, Benjamin, New York, 1967. 
3. B. Blackadar and D. Handelman, Dimension functions and traces on $C^{*}$-algebras, J. Funct. Anal. 45 (1982), 297-340.

4. M. Breuer, On the homotopy type of the group of regular elements of semifinite von Neumann algebras, Math. Ann. 185 (1970), 61-74.

5. J. Brüning and W. Willgerodt, Eine Verallgemeinerung eines Satzes von N. Kuiper, Math. Ann. 222 (1976), 47-58.

6. R. C. Busby, Double centralizers and extensions of $C^{*}$-algebras, Trans. Amer. Math. Soc. 132 (1968), 79-99.

7. A. Connes, An analogue of the Thom isomorphism for crossed products of a $C^{*}$-algebra by an action of R, Adv. in Math. 39 (1981), 31-55.

8. E. G. Effros and E. C. Lance, Tensor products of operator algebras, Adv. in Math. 25 (1977), 1-34.

9. G. A. Elliott, Derivations of matroid $C^{*}$-algebras. II, Ann. of Math. (2) 100 (1974), 407-422.

10. K. R. Goodearl and D. Handelman, Stenosis in dimension groups and $A F C^{*}$-algebras, J. Reine Agnew. Math. 332 (1982), 1-98.

11. G. G. Kasparov, Hilbert $C^{*}$-modules; Theorems of Stinespring and Voiculescu, J. Operator Theory 4 (1980), 133-150.

12. The operator K-functor and extensions of $C^{*}$-algebras, Izv. Akad. Nauk SSSR 44 (1980), 571-636; English transl., Math. USSR Izv. 16 (1981), 513-572.

13. N. Kuiper, The homotopy type of the unitary group of Hilbert space, Topology 3 (1965), 19-30.

14. J. A. Mingo, On the contractibility of the unitary group of the Hilbert space over a $C^{*}$-algebra, J. Integral Equations Operator Theory 5 (1982), 888-891.

15. A. S. Miščenko and A. T. Fomenko, The index of elliptic operators over $C^{*}$-algebras, Izv. Akad. Nauk SSSR 43 (1979), 831-859; English transl., Math. USSR Izv. 15 (1980), 87-112.

16. G. K. Pedersen, $C^{*}$-algebras and their automorphism groups, Academic Press, London, 1979.

17. M. Pimsner, S. Popa and D. Voiculescu, Homogeneous extensions of $C(X) \otimes K(H)$. II, J. Operator Theory 4 (1980), 211-249.

18. G. Segal, Equivariant contractibility of the general linear group of Hilbert space, Bull. London Math. Soc. 1 (1969), 329-331.

19. J. Taylor, Banach algebras and topology, Algebras and Analysis (J. H. Williamson, ed.), Academic Press, London, 1975.

Department of Mathematics, University of Toronto, Toronto, Ontario, Canada M5S 1A1 